Working Paper 11-13

Economic Series

June, 2011
Departamento de Economía Universidad Carlos III de Madrid Calle Madrid, 126 28903 Getafe (Spain) Fax (34) 916249875

\title{
Endogenous Strength in Conflicts
}

\author{
Carmen Beviá \\ Departament d'Economia i d'Història Econòmica \\ Universitat Autònoma de Barcelona
}

\author{
Luis C. Corchón \\ Departamento de Economía \\ Universidad Carlos III
}

First version October 2010. This version June 13th, 2011

\begin{abstract}
In this paper we study a two stage contest where the strength of players in the second stage depends on the result of the contest in the first stage. We show that this contest displays properties that are not present in one shot contests. Non-symmetric players make different efforts in the first stage and rent dissipation in the first period may be large. We study the conditions under which the discouragement effect holds. In addition, new issues emerge like the evolution of the strengths and the shares of the prize during the game.
\end{abstract}

\footnotetext{
* We thank M. Dahm, A. Heifetz, M. Kolmar, M. Kredler, C. Ponsati, S. Skaperdas, A. Wolinsky, A. Yildizparlak and the participants in a seminar at U. Carlos III, the second congress on Tournaments, Contests and Relative Performance Evaluation, U. of North Carolina, the meeting "Games and Social Decisions", Northwestern U. and the workshop on "Games with an Aggregative Structure", U. of Strathclyde for very interesting coments on earlier versions of the paper. The first author acknowledges financial support from ECO2008-04756 (Grupo ConsolidadoC), SGR2009-419, Barcelona GSE research network and MOVE where she is an affiliated researcher. The second author acknowledges financial support from SEJ2005-06167/ECON.
} 


\section{Introduction}

The theory of contests analyzes situations in which several contenders expend effort to win a prize. The theory developed from the initial papers by Tullock (1967), Krueger (1974) and Becker (1984), see also Hirshleifer (1991), assumed in the main that the effort of different players had an identical impact in the contest. We will refer to this impact as the strength of a player. Static models in which players have different strengths were considered by Hillman and Riley (1989), Gradstein (1995), Corchón (2000) and Cornes and Hartley (2005).

Dynamic contests have been studied in a number of papers focussing on infinite horizon models (Cairns, 1989; Wirl, 1994; Leininger and Chun-Lei, 1994; and McBride and Skaperdas, 2007), two period models of war and settlement (Skaperdas and Syropoulos, 1996; Skaperdas and Garfinkel, 2000) and models in which players have to win a number of contests in order to win a grand contest (Konrad and Kovenock, 2009; see also the surveys of Konrad, 2009, Chpt. 8, and Konrad, 2010). All these papers assume that the strength of players does not vary during the contest.

In this paper we present a two period, two players contest in which the strength of players is endogenous. We assume that the strength of a player in the second period depends on the result of the contest in the first period. Think of a war composed of two battles where the outcome of the first battle determines the strength of players in the second battle. This is a general idea that we want to explore in a particular setting in which the strength of contenders in the second period depends on the share of the prize obtained in the first period (we assume that the prize is divisible). This assumption captures situations such as wars in which the strength of a country depends on the fraction of the territory owned by this country. Another example might be the cold war between the USSR and the US in which the relative strength of each side could be measured by the territories (or the population) under its control.

The model is spelled out in Section 2. We assume that the transition function is a concave contraction and costs are linear on effort. The contest success function (CSF) is assumed to be of a generalized Tullock form in which the efforts of players are raised to an number between zero and one and the resultant numbers are weighted by their strengths. We assume that the only source of asymmetry between players is their strength. This assumption is made in order to focus our study on the impact of initial strength on the equilibrium outcome of the game.

In Section 3 we prove the existence of a Subgame Perfect Nash Equilibrium (Proposition 1). 
This equilibrium displays features different from those in one shot models: Non-symmetric players make different efforts in the first period and, consequently, the share of the prize in the hands of a player in the first period does not equal her relative strength. We also prove that when the exponent of effort is one and the transition function is linear the equilibrium is unique (Proposition 2 ). These two assumptions will be maintained in the rest of the paper.

In Section 4 we explore the properties of equilibrium. We first prove some properties of equilibrium efforts with respect to the initial relative strength of player 1 . We show that the effort of a player is a mirror image of the effort of the other player (since the only source of asymmetry is the relative strength) and that the player with the largest relative strength makes the larger effort (Proposition 3). The latter is not true in one shot games with two players and Tullock CSF. Relative strengths count here because the second period creates different incentives for players with different relative strengths. We show that the ratio of the effort of player 1 with respect to player 2 in period 1 is increasing in the relative strength of player 1 (Proposition 4). Thus, when the effort in the first period is also an investment for the second period, the stronger player makes more effort in both absolute and relative terms than the weaker player.

The previous properties prompt us to compare the effort made in the first period of our game with the effort made if the game were one shot. This has been studied in several papers and discussed in Konrad (2010). In many cases, multi-stage contests involve a "discouragement effect" in which weak players make less effort in early stages than they would if the contest were one shot. We find that the discouragement effect also holds in our framework when the weak player is sufficiently weak (Proposition 5). But it does not always hold. Even if a player is three times stronger than the other the latter makes more effort than in a one shot game. This is because in our framework agents receive a prize in each period and not only at the end of the grand contest.

Next we study the trajectory of relative strengths during the game. Clearly, the relative strength of a player in the second period is an increasing function of the relative strength of this player at the start of the game. When the link between periods is strong (no discount and the strength in the second period equals the share in the prize in the first period) an initially strong (resp. weak) player will be even stronger (weaker) in the second period. We call this the "avalanche effect" because the initial advantage of a player is amplified later on. However when the link between periods is not strong the avalanche effect only occurs when initial strengths are similar. When initial strengths 
are unequal the relative strength of the strong player decreases in the second period. We call this the "level-off" effect. It is caused by an increase in the relative effort of the weak player. When the link between periods is weak the avalanche effect disappears, so in the second period relative strengths are levelled off with respect to what they were in the first period. The range of parameters for which both effects occur is characterized in Proposition $6 .^{1}$

Finally we study rent dissipation. We show that only when players have identical initial strengths and the link between periods is the strongest, rents are completely dissipated (Proposition 7). When players are very similar and the link between periods is strong, there is more rent dissipation in the two stage game than in the one shot game (Proposition 8). But rent dissipation is not monotonic with the link between periods; thus weak links can be associated with more rent dissipation than strong links. This is due to the discouragement effect.

Finally Section 5 offers our closing comments.

Our paper is related to the literature providing foundations to CSF. In some papers the CSF is determined by a planner. We do not review this literature but note that the closest papers to ours in terms of goals are by Nti (2004) and Franke et alia (2009) who look for the optimal weights of a given CSF. In other papers the CSF is not determined by a planner. We call these situations conflicts. Fearon (1996) (see also Leventoglu and Slantchev, 2007) presented a model in which the bargaining power is endogenous and determined by the size of the territory and the threat of a war in which one of the countries would disappear. In our model there is neither bargaining nor final war but a protracted conflict like in the multi-batle models mentioned before.

The closest paper to ours is by Klumpp and Polborn (2006). In their model, candidates to office have to win a certain number of elections in order to win the grand contest. They show that the outcome of the first election creates an asymmetry in later rounds which might be decisive for the grand contest. Thus they provide an explanation based on rational agents for the "momentum effect" which is the tendency of early winners in preliminary contests to win the grand contest. The main difference with our paper is that the prize is obtained at the end of the grand conflict and that the strengths of players is exogenous. In their case the expected value of the prize at each moment is the variable which changes as the game is unfolding.

\footnotetext{
${ }^{1}$ We also study the trajectory of the share of the prize in the hands of player 1.
} 


\section{The Model}

\subsection{Players and payoffs}

Two players, $i \in\{1,2\}$, fight for a divisible prize in two periods, $t \in\{1,2\}$. Each player ends each period with a fraction $p_{i}^{t}$ of the prize. The value of the prize for each player in each period is $V$. The interpretation is that the resource under conflict produces a certain surplus each period that can be expropriated by the owner (harvest, money, slave population, human capital, etc.) and that this surplus does not depend on the intensity of conflict.

Player $i$ makes an effort $e_{i}^{t}$ in period $t$. We assume that the marginal cost of effort is constant and equal to 1 . Payoffs in period $t$ are denoted by $\pi_{i}^{t}$ and equal $p_{i}^{t} V-e_{i}^{t} i=1,2$. Payoffs for the whole game are $\sum_{t=1}^{2} \delta^{t-1} \pi_{i}^{t} \equiv \Pi_{i}$ where $\delta \in[0,1]$ is the discount rate of the players.

Players have relative strengths which determine the impact of their effort. We denote by $\alpha^{t} \in[0,1]$ the relative strength of player 1 at $t$, and by $1-\alpha^{t}$ the relative strength of player 2 at $t$. The contest success function (CSF) maps efforts and strengths in a period into the fraction of the price owned by the players in this period. Let $p$ (resp. $1-p$ ) be the fraction obtained by player 1 (resp. 2). We assume the CSF takes the asymmetric general Tullock (1980) form:

$$
\begin{aligned}
p^{t} & =\frac{\alpha^{t}\left(e_{1}^{t}\right)^{\gamma}}{\alpha^{t}\left(e_{1}^{t}\right)^{\gamma}+\left(1-\alpha^{t}\right)\left(e_{2}^{t}\right)^{\gamma}} \text { if } e_{1}^{t}+e_{2}^{t}>0 ; \quad p^{t}=\alpha^{t} \text { otherwise. } \\
1-p^{t} & =\frac{\left(1-\alpha^{t}\right)\left(e_{2}^{t}\right)^{\gamma}}{\alpha^{t}\left(e_{1}^{t}\right)^{\gamma}+\left(1-\alpha^{t}\right)\left(e_{2}^{t}\right)^{\gamma}} \text { if } e_{1}^{t}+e_{2}^{t}>0 ; \quad 1-p^{t}=1-\alpha^{t} \text { otherwise. }
\end{aligned}
$$

Thus, efforts and relative strength enter multiplicatively in the CSF. Think of the relative strength as capital (social or physical) or territory and of $\alpha^{t}\left(e_{i}^{t}\right)^{\gamma}$ as the (Cobb-Douglas) production function of the influence of player $i$ in the contest. Thus influence in the contest is produced by capital and labor. The parameter $\gamma$ measures the sensitivity of the probability of winning to the efforts. When $\gamma=0$, the outcome of the contest is independent of efforts. When $\gamma=1$, the CSF is proportional. It seems reasonable to require that the CSF is homogeneous of degree zero, so winning probabilities do not depend on how resources are measured (euros or dollars, thousands or millions of soldiers, etc.).

Clark and Riis (1998), following Skaperdas (1996), have shown that under certain assumptions the only functional form that is homogeneous of degree zero is precisely the one above.

Finally, note that the only source of asymmetry among players in payoffs and strategies comes from relative strength in period one which is exogenously given. 


\subsection{The transition function}

The relative strength of player 1 changes from period one to period two according to the following transition function:

$$
\alpha^{t}=f\left(p^{t-1}\right),
$$

where $f:[0,1] \rightarrow[0,1]$ fulfils the following properties:

i) $f(1 / 2)=1 / 2$,

ii) $f(p) \leq p$ if $p \geq 1 / 2, f(p) \geq p$ if $p \leq 1 / 2$, for all $p \in[0,1]$,

iii) $0<f^{\prime}(p) \leq 1, f^{\prime \prime}(p) \leq 0$, for all $p \in[0,1]$.

Properties i) and ii) reflect the symmetry of the transition function. This plus the assumptions made in the previous subsection allows us to identify $\alpha$ as the unique source of asymmetry in our model. Thus, the impact of initial strength on efforts will be the main theme of this paper. In order to motivate ii) and iii) consider the following example of a linear transition function:

$$
f\left(p^{t-1}\right)=a p^{t-1}+b, \text { with } 0<a \leq 1, a=1-2 b, b \geq 0,
$$

where $a$ measures the importance of the share of the resource in the previous period and $b$ the strength of country 1 which does not depend on the share. Since $a>0$ the ownership of the resource contributes positively to the relative strength, i.e. more people to draft or more/better sources of food, money, etc. The condition $a=1-2 b$ makes $p$ and $1-p$ symmetric because the strength for player 2 evolves according to

$$
1-\alpha^{t}=1-a p^{t-1}-b=a\left(1-p^{t-1}\right)+1-a-b .
$$

It seems natural to assume that even if a country has a zero share in the resource it has a nonnegative relative strength. Thus,

$$
b \geq 0 \quad \text { and } \quad 1 \geq a+b .
$$

It is also natural to assume that the relative strength of a country is not maximal when it owns zero of the resource. Thus

$$
b \leq 1 \quad \text { and } \quad b+a \geq 0 .
$$

Conditions (2.6) and (2.7) imply $a \in[0,1]$ which corresponds to $f(p) \leq p$ if $p \geq 1 / 2$ (and the symmetric property when $p<1 / 2), f^{\prime}(p) \leq 1, f^{\prime \prime}(p) \leq 0$. 


\section{Equilibrium}

We look for a Subgame Perfect Nash Equilibrium of the game described in the previous section. Since there are only two periods, the game is solved backwards.

In what follows and in order to simplify notation we will denote with prime the variables in the second period and without prime the variables in the first period.

In the second period, since the game ends, players play the one shot Nash equilibrium. Thus,

$$
e_{1}^{\prime}=e_{2}^{\prime}=\gamma\left(1-\alpha^{\prime}\right) \alpha^{\prime} V
$$

and the fraction of the price that agent 1 gets in the second period is given by:

$$
p^{\prime}=\alpha^{\prime}=f(p)
$$

Payoffs in the second period, given (3.2), are:

$$
\begin{aligned}
\pi_{1}^{\prime} & =f(p) V-\gamma f(p)(1-f(p)) V= \\
& =f(p) V(1-\gamma(1-f(p))) . \\
\pi_{2}^{\prime}= & (1-f(p)) V-\gamma f(p)(1-f(p)) V= \\
= & (1-f(p)) V(1-\gamma f(p)) .
\end{aligned}
$$

In the first period, each player solves:

$$
\begin{gathered}
\max _{e_{1}} p V-e_{1}+\delta f(p) V(1-\gamma(1-f(p))) \\
\max _{e_{2}}(1-p) V-e_{2}+\delta(1-f(p)) V(1-\gamma f(p))
\end{gathered}
$$

First order conditions of payoff maximization for both players are:

$$
\begin{aligned}
& \frac{\partial p}{\partial e_{1}} V\left[1+\delta f^{\prime}(p)(1-\gamma+2 \gamma f(p))\right]=1, \\
& -\frac{\partial p}{\partial e_{2}} V\left[1+\delta f^{\prime}(p)(1+\gamma-2 \gamma f(p))\right]=1 .
\end{aligned}
$$

In the Appendix we show that the second order conditions of payoff maximization are fulfilled. Note first that $p$ is as a function of relative efforts and relative strengths. Let $x=e_{1} / e_{2}$. And let $h_{1}(\cdot, \cdot)$ and $h_{2}(\cdot, \cdot)$ be defined as follows

$$
h_{1}(x, \alpha)=1+\delta f^{\prime}(p)(1-\gamma+2 \gamma f(p)),
$$




$$
h_{2}(x, \alpha)=1+\delta f^{\prime}(p)(1+\gamma-2 \gamma f(p))
$$

Thus, the first order conditions can be rewritten as:

$$
\begin{aligned}
& \frac{\gamma \alpha(1-\alpha) e_{1}^{\gamma-1} e_{2}^{\gamma}}{\left(\alpha e_{1}^{\gamma}+(1-\alpha) e_{2}^{\gamma}\right)^{2}} V h_{1}(x, \alpha)=1 \\
& \frac{\gamma \alpha(1-\alpha) e_{1}^{\gamma} e_{2}^{\gamma-1}}{\left(\alpha e_{1}^{\gamma}+(1-\alpha) e_{2}^{\gamma}\right)^{2}} V h_{2}(x, \alpha)=1 .
\end{aligned}
$$

Thus,

$$
e_{2} h_{1}(x, \alpha)=e_{1} h_{2}(x, \alpha)
$$

Dividing the above equation by $e_{2}$ we get:

$$
h_{1}(x, \alpha)-x h_{2}(x, \alpha)=0
$$

We show in the Appendix that the above equation has a solution. Let $x=x(\alpha)$ be one of the solutions of this equation. Thus, from equation (3.13) we get that

$$
\begin{aligned}
& e_{1}(\alpha)=\frac{\gamma \alpha(1-\alpha)(x(\alpha))^{\gamma}}{\left(\alpha(x(\alpha))^{\gamma}+(1-\alpha)\right)^{2}} V h_{1}(x(\alpha), \alpha) ; \\
& e_{2}(\alpha)=\frac{\gamma \alpha(1-\alpha)(x(\alpha))^{\gamma-1}}{\left(\alpha(x(\alpha))^{\gamma}+(1-\alpha)\right)^{2}} V h_{1}(x(\alpha), \alpha) .
\end{aligned}
$$

which are the equilibrium efforts. Thus we have shown,

Proposition 1. A Subgame Perfect Nash Equilibrium exists.

Note that periods are linked by the discount rate $\delta$ and the the transition function. When $\delta$ is zero, or the transition function is constant, this link is severed and our equilibrium is just the one shot equilibrium. This is seen from (3.11) and (3.12) because then $h_{1}(x, \alpha)=h_{2}(x, \alpha)=1$ so $x(\alpha)=1$ and efforts in (3.17) and (3.18) collapse in the one shot equilibrium values which are

$$
e_{1}^{o s}(\alpha)=e_{2}^{o s}(\alpha)=\gamma \alpha(1-\alpha) V
$$

Even if the strength of players is different, the effort made in equilibrium in the one shot game is the same for both players. This property holds as long as there are two players with identical valuations and the CSF is homogeneous of degree zero (Corchón 2000). In our two stage game this 
property does not hold in the first period, reflecting the different strategic opportunities for both players in the continuation game.

In general, we can not guarantee uniqueness of equilibrium. Uniqueness is obtained if the transition function is linear and the contest success function is proportional to weighted efforts. We formally state this in the following proposition. The proof is in the Appendix.

Proposition 2. If $\gamma=1$ and $f(p)$ is linear, there exist a unique Subgame Perfect Nash Equilibrium.

To close this section, note that in the case described in Proposition 2, plugging (3.17) and (3.18) in (2.1) we obtain that the fraction of the resource owned by player 1 in period 1 is

$$
p(\alpha)=\frac{\alpha x(\alpha)}{\alpha x(\alpha)+1-\alpha} .
$$

It is easy to show that since $x(\cdot)$ is increasing (see Proposition 4 below) $p(\cdot)$ is increasing. So, as in the one shot game -where $p(\alpha)=\alpha$ - the fraction of resources owned by player 1 in period 1 depends positively on the initial strength (as intuition suggests), though in a more complicated way.

Finally, notice that $V$ does not affect the equilibrium distribution of the prize between players in both periods. This also happens in the one shot game.

In what follows we restrict the analysis of the properties of equilibrium to the special case described in Proposition 2. This assures uniqueness of equilibrium which seems a sensible requirement when exploring the properties of equilibrium.

\section{Properties of equilibrium}

\subsection{Preliminary properties}

We first state and prove some properties of equilibrium efforts that will be useful later on. We will see that some of these properties differ from the corresponding properties in a one shot game. All the proofs are gathered in the Appendix.

Proposition 3. The equilibrium efforts in the first period satisfy the following:

(i) $e_{2}(\alpha)=e_{1}(1-\alpha)$;

(ii) $e_{1}(\alpha)=e_{2}(\alpha)$ for $\alpha=1 / 2, \alpha=0, \alpha=1$;

(iii) $e_{1}(\alpha)>e_{2}(\alpha)$ if and only if $\alpha>1 / 2$. 
Proposition 3 says that individual efforts display symmetry properties inherited from the symmetry of the basic data of the problem. Part (i) says that the effort of player 1 is the mirror image of the effort of player 2 when her relative strength $\alpha$ is substituted by $1-\alpha$. Part (ii) says that the effort of both players are identical either when they have the same relative strength $(\alpha=1 / 2)$ or when one of them has zero strength. Part (iii) says that the player with larger strength makes larger effort. Notice that this is not true in the one shot game, so this fact is explained by the existence of a second period.

The next result studies the ratio of efforts.

Proposition 4. The ratio of the equilibrium efforts in the first period, $x(\alpha)$, is increasing in $\alpha$.

Proposition 4 says that relative efforts are increasing with relative strength. Thus, the strong player makes more effort in the first period than the weaker player, which leaves her in better shape for the conflict in the second period. This contrasts with the one shot game where $x(\alpha)=1$ for all $\alpha \in[0,1]$.

\subsection{The Discouragement effect}

We now address the question of when agents make more effort in our two stage game than in the one shot game. We start by considering the following example.

Example 1. Suppose that $a=1, b=0$ and $V=10$. In this case we obtain a closed form solution for efforts and $x$, namely

$$
x(\alpha)=\frac{2 \alpha-2 \delta+4 \alpha \delta-1+\sqrt{4 \delta+4 \delta^{2}+16 \alpha^{2} \delta^{2}-16 \alpha \delta-16 \alpha \delta^{2}+16 \alpha^{2} \delta+1}}{2 \alpha} .
$$

In Figure 1 below we show the effort in the first period for both players as a function of $\alpha$. We

draw the case of $\delta=1$. The solid line corresponds to player 1 and the dashed line to player 2 . Note the symmetry of the two lines, as proved in 3 part i). The dotted line corresponds to the effort of each player in the one shot game. 


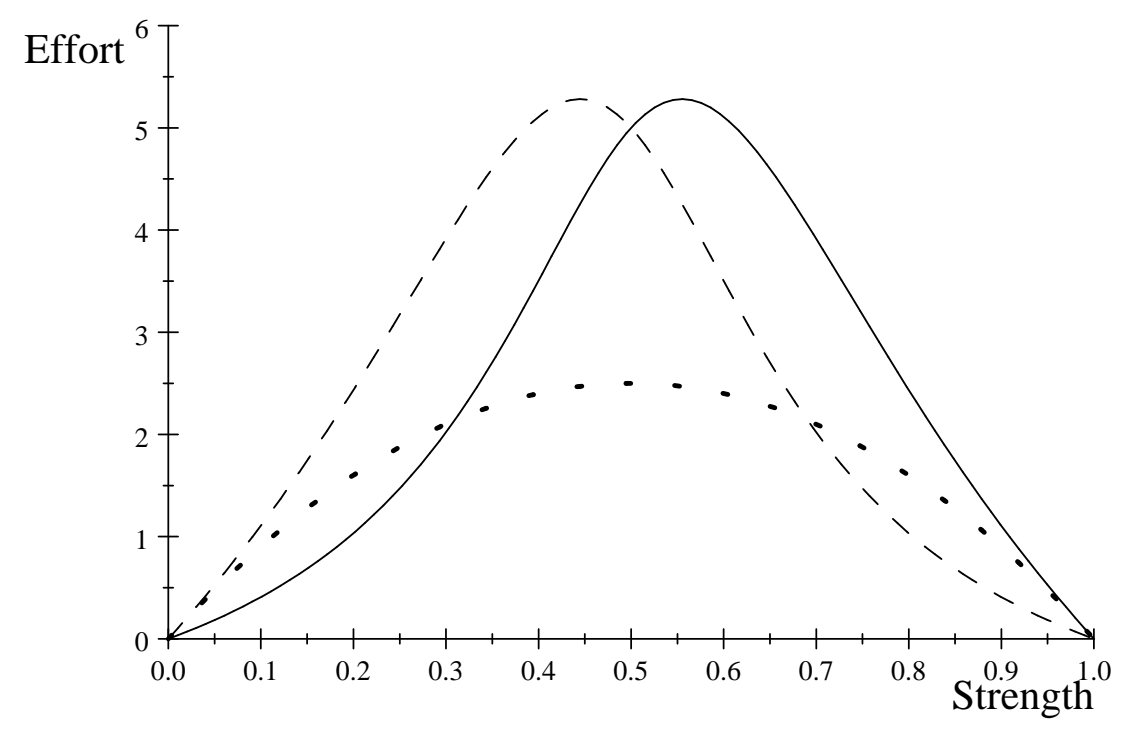

Figure 1

When the strength of a player is very large or very small, this player exerts little effort. This is because the outcome of the contest is very biased on -or against- her. When the contest is "fair" in the sense that similar efforts have similar impacts on the contests, efforts are larger.

We can see the effect of introducing a second period. If a player has little strength (approximately less than .3 in the figure for player 1) she is discouraged by the existence of a second period in the sense of exerting less effort in the two-period game than in the one period game. However, for larger values of strength, the existence of a second period encourages players to exercise more effort than in the one period game.

The example above exhibits a "discouragement effect" which is when weak players "reduce their incentives to expend effort in early rounds," (Konrad and Kovenock, 2010, p. 95 see the references there for earlier analysis of this effect and Konrad (2009, pp. 189-191) for a survey). This effect runs counter to the intuition that in a multiperiod game, players exert more effort than in a single period game because each period adds more return to the effort and thus incentives to expend more effort are enhanced by the existence of additional periods. This intuition is correct when first order conditions of payoff maximization are unaffected by the effort of other players. But when this is not the case the situation might be reversed. The next Proposition analyzes this effect for player 1. The analysis for player 2 would be totally symmetric. 
Proposition 5. If $a, \delta>0$, there exist $\alpha^{*} \in(0,1 / 2)$ such that for all $\alpha \in\left(0, \alpha^{*}\right)$, the equilibrium effort of player 1 in period 1 is smaller than the equilibrium effort in the one shot game.

This result says that the discouragement effect happens when one of the players is sufficiently weak. But as Figure 1 makes clear, even for reasonably low values of the strength of the weak player, say $\alpha=1 / 3$, the discouragement effect does not hold.

The discouragement effect is less and less severe as $a$ or $\delta$ becomes smaller. In the limit case ( $a=0$ or $\delta=0$ ) the effect disappears because the equilibrium values of efforts collapse in the value corresponding to the one shot equilibrium. In Figure 2 we represent the effort of player 1 for different values of $a$ and $\delta=1$. The solid line corresponds to $a=1$, the dashed line corresponds to $a=0.8$, and the dotted line corresponds to the one shot game which is equal to $a=0$. Similar effects are obtained when $\delta$ decreases.

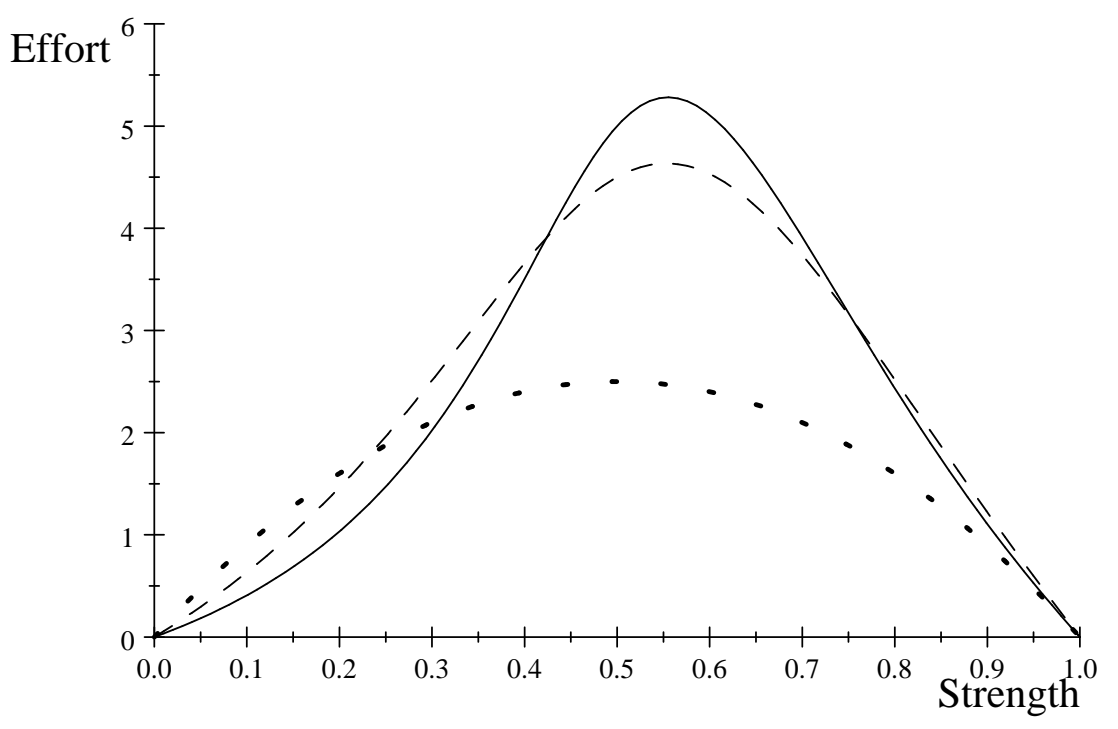

Figure 2

\subsection{Avalanches or level off?}

The second question that we address is the trajectory of strengths. Since $\alpha^{\prime}$ is increasing in $p$ which in turn is increasing in $\alpha$, it follows that $\alpha^{\prime}$ is increasing in $\alpha$. But this does not imply anything about whether $\alpha \gtrless \alpha^{\prime}{ }^{2}$

\footnotetext{
${ }^{2}$ Clearly, if $\alpha=1 / 2, \alpha^{\prime}=1 / 2$ too.
} 
A possibility is that when player 1 is initially strong $(\alpha>1 / 2)$ she will be even stronger in the second period $\left(\alpha^{\prime}>\alpha\right)$. We call this situation the avalanche effect of the second period because the strength of strong (resp. weak) players is amplified. ${ }^{3}$ We see that this is the case when $a=\delta=1$ and $b=0$. This follows from the fact that $\alpha^{\prime}=\alpha x /(\alpha x+1-\alpha)$ is increasing in $x$ and for $\alpha>1 / 2$, $x>1$. This is represented in Figure 3 below by a sinusoid solid line. The straight solid line is the $45^{0}$ line.

But when $a=0.8, \delta=1$ and $b=0.1$-represented in Figure 3 by the dotted line- this line intersects the $45^{0}$ line in three points. From $1 / 2$ to the intersection to the right of $1 / 2$ (or from the intersection to the left of $1 / 2$ to zero) the avalanche effect still holds. However for $\alpha$ close to one, $\alpha^{\prime}<\alpha$ and for $\alpha$ close to zero $\alpha^{\prime}>\alpha$. Thus the existence of a second period levels off relative strengths.

Finally, the dashed line in Figure 3 represents the case $a=0.5, \delta=1$ and $b=0.25$. In this case the avalanche effect disappears completely and starting from any position the relative strength of players is levelled off in the second period.

In fact these three cases exhaust all the possibilities that might arise in our framework. This is shown in the next proposition where the case a) corresponds to the solid line, the case $b$ ) corresponds to the dotted line and the case $c$ ) corresponds to the dashed line in Figure 3.

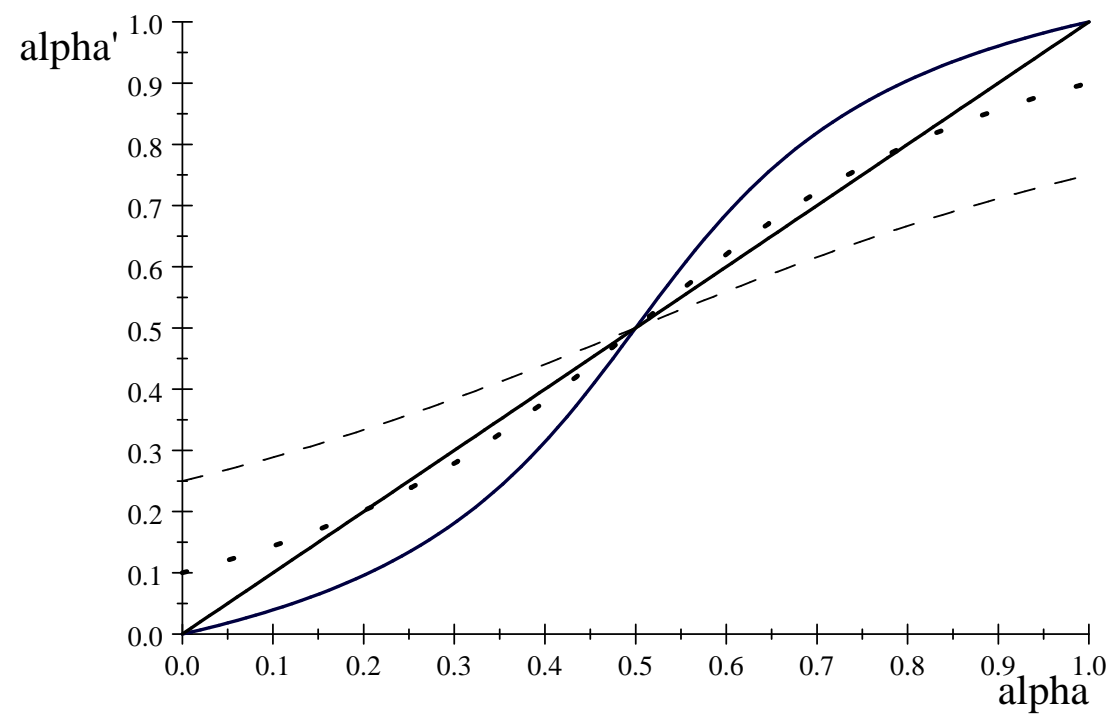

Figure 3

\footnotetext{
${ }^{3}$ This effect has consequences similar to the momentum effect in Klumpp and Polborn (2006). But the momentum effect operates through the value of the prize and the avalanche effect through the strength of players.
} 
Proposition 6. a) If $b=0$ there is an avalanche effect for all $\alpha \in[0,1] \backslash\{1 / 2\}$.

b) If $0<b<1 / 4$ and $\delta>2 b /(1-2 b)(1-4 b)$, there exist $\bar{\alpha}<1 / 2$ (resp. $\hat{\alpha}>1 / 2)$ such that for all $\alpha \in(0, \bar{\alpha})$ (resp. $\alpha \in(\hat{\alpha}, 1))$ there is a level-off effect. For all $\alpha \in(\bar{\alpha}, 1 / 2)$ (resp. $\alpha \in(1 / 2, \hat{\alpha}))$ there is an avalanche effect.

c) If $0<b<1 / 4$ and $\delta<2 b /(1-2 b)(1-4 b)$, or if $b \geq 1 / 4$ and $\delta \in[0,1]$ there is a level-off effect for all $\alpha \in[0,1]$ except, possibly, for two isolated values of $\alpha$.

The condition $\delta>2 b /(1-2 b)(1-4 b)$ and $b<1 / 4$ is equivalent to $d \alpha^{\prime} / d \alpha>1$ at $\alpha=1 / 2$. In this case the curve relating $\alpha$ and $\alpha^{\prime}$ crosses the $45^{\circ}$ from below like the solid line (where $d \alpha^{\prime} / d \alpha=2$ at $\alpha=1 / 2$ ) and the dotted line (where $d \alpha^{\prime} / d \alpha=1.2414$ at $\alpha=1 / 2$ ) in Figure 3. Finally, the conditions $b<1 / 4$, and $\delta<2 b /(1-2 b)(1-4 b)$, or $b \geq 1 / 4$ and $\delta \in[0,1]$ implies that $d \alpha^{\prime} / d \alpha<1$ at $\alpha=1 / 2$ like the dashed line in Figure 3 (where $d \alpha^{\prime} / d \alpha=0.6$ at $\alpha=1 / 2$ ).

\subsection{The domino effect}

The third question that we address is the trajectory of the share of the prize in the hands of player 1. This share summarizes the equilibrium outcome of our game. One would expect that this share follows the behavior of $\alpha$. We see that this is not the case.

Following the ideas introduced in the previous subsection consider the possibility that when player 1 is having initially more than half of the prize $(p>1 / 2)$ she will have even a larger share in the second period $\left(p^{\prime}>p\right)$. We call this situation the domino effect of the second period because the initial share of a strong (resp. weak) player is amplified later on in the game. Notice that

$$
p^{\prime}=a p+b=(1-2 b) p+b
$$

Rearranging (4.2) we obtain

$$
p^{\prime}-p=b(1-2 p) .
$$

Thus we have two cases. In the extreme case in which only the outcome in the first period is relevant to determine the strength next period (i.e. $b=0$ ), $p^{\prime}=p$ so shares are invariant in time. In any other case, $b>0$ and $p>1 / 2$ implies $p^{\prime}<p$, irrespective of whether there is an avalanche or a level off effect. This suggests that protracted conflicts tend to end up in an impasse in which players have to spend resources period after period in order to maintain their position. Examples like the Roman empire vs. Germanic tribes or vs. the Persian Empire, the first World War (until 
the entry of US in the conflict) or the cold war come to our mind. However, a full proof of this conjecture would take a model with several periods which is not attempted here. We do not enter in the discussion of what kind of modelling is preferable, a two stage model or an infinite horizon model. For an enthusiastic defense of the former see Shapiro (1989).

\subsection{Rent Dissipation}

Our final question is the impact of the second period on the rent dissipation in the first period. In the second period since efforts equal those in a one shot game rent dissipation is like in a one shot game.

Total effort in the first period amounts to

$$
\frac{\alpha(1-\alpha) x(\alpha)}{(\alpha x(\alpha)+(1-\alpha))^{2}} V h_{1}(x(\alpha), \alpha)+\frac{\alpha(1-\alpha)}{(\alpha x(\alpha)+(1-\alpha))^{2}} V h_{1}(x(\alpha), \alpha) .
$$

Since in equilibrium $h_{1}(x(\alpha), \alpha)=x(\alpha) h_{2}(x(\alpha), \alpha)$, and $h_{1}(x(\alpha), \alpha)+h_{2}(x(\alpha), \alpha)=2(1+\delta a),(4.4)$ can be written as:

$$
\frac{\alpha(1-\alpha) x(\alpha) 2(1+\delta a) V}{(\alpha x(\alpha)+(1-\alpha))^{2}} .
$$

Call this function $B(\alpha, d, x)$ where $d \equiv \delta a$. We now study the maxima of $B()$ with respect to $\alpha, d$ and $x$. Given that $B()$ does not take into account the dependence of $x$ with respect to the other variables, the maxima of $B()$ is always larger or equal than the maximum amount of effort. We see immediately that $B()$ is increasing in $d$ so in the maximum $d=1$ (which implies that $a=\delta=1$ ). We also see that the maximum with respect to $\alpha$ cannot be at the boundaries of $[0,1]$ because there, the function takes the value 0 . Also, the maximum cannot be at either $x=0$ (where the function takes the value 0) or at an arbitrarily large value of $x$ where the function takes a value arbitrarily close to 0 . Thus the maximum with respect to $\alpha$ and $x$ must be interior. Computing

$$
\begin{aligned}
& \frac{\partial B(\alpha, d, x)}{\partial \alpha}=0 \text { yields } \alpha=\frac{1}{x+1} \\
& \frac{\partial B(\alpha, d, x)}{\partial x}=0 \text { yields } x=\frac{1-\alpha}{\alpha} .
\end{aligned}
$$

Equations (4.6) and (4.7) are identical so there is a continuum of solutions. We now introduce the fact that $x$ is increasing in $\alpha$ and it is always positive. Thus $1 /(x(\alpha)+1)$ is decreasing in $\alpha$ and strictly positive. So (4.6) has a unique solution. Nothe that for $\alpha=1 / 2, x(\alpha)=1$, and this is always a solution of (4.6). So, this must be the unique solution. We have proved the following. 
Proposition 7. Rents are completely dissipated iff $\alpha=1 / 2, \delta=a=1$.

The previous result calls for a comparison of the rent dissipation in our game and in the one shot game. In the latter total efforts are

$$
2 \alpha(1-\alpha) V
$$

In this case, rents are never completely dissipated. Thus we have the following

Proposition 8. For $\alpha$ close to $1 / 2$ and $\delta$ and a close to 1 , there is more rent dissipation in the two stage game than in the one shot game.

The result follows from the fact that the correspondence mapping $\alpha, \delta$ and $a$ into efforts has a closed graph in $(0,1) \times[0,1] \times[0,1]$. Since this correspondence is a function (because equilibrium is unique) this function is continuous and the result follows. ${ }^{4}$

Thus, when the link between periods is stronger (no discount and strengths are derived directly from the share in the first period) competition among agents dissipates the prize entirely. In this case competition is tougher because to the effect of fighting for the price in the first period, we have to add the effect of maintaining relative strengths in the second period. Clearly, as strength in the second period depends less on effort in the first period, this second effect vanishes. In Figure 5 we show how total effort in the first period changes with $a$ for the case of $\delta=1$. The solid line corresponds to $a=1$, the dashed line corresponds to $a=0.5$, and the dotted line corresponds to $a=0$. We note that, due to the discouragement effect, in some cases, conflict is less severe than in the one shot game.

\footnotetext{
${ }^{4}$ Notice that this result cannot be obtained directly from the function $B()$ since this function does not incorporate the restriction that $x=x(\alpha)$.
} 


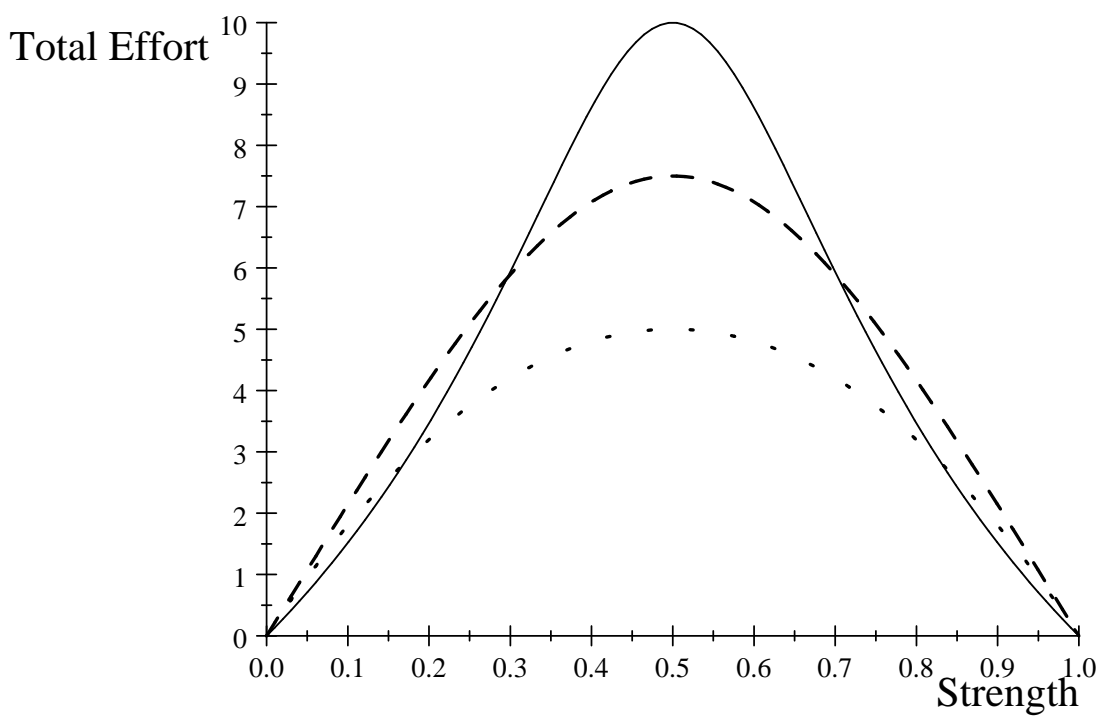

Figure 5

\section{Final comments}

In this paper we have developed a theory of endogenous strength. We assumed that the strength in a period is a function of the fraction of the resource enjoyed by a player. We have found that equilibrium displays some features different from the one shot game. In particular rents might be completely dissipated in the first period and players with different strengths make different efforts in the first period. Our model also differs from other multi-contest models in which the discouragement effect is pervasive. Finally new issues appear like the avalanche/level-off effect and the domino effect.

In order to get a tractable model, we assume two players, two periods and a linear transition function. The assumption that $a \leq 1$ plays also an important role in our proofs. Therefore, it would be convenient to investigate a model in which $a>1$ or in which the transition function is not always increasing reflecting that too much territory might be disadvantageous for strength. But this is outside the scope of this paper. Here we try to make a first cut in the issue of the evolution of strength when it depends on past outcomes. Our conclusions are, of course, tentative.

Our model does not pay attention to issues which play an important role in dynamic conflicts. Among them we note the following two.

1. There are no resource constraints in the model. Consequently there are no bankruptcies. 
But the history of Europe has plenty of examples where conflict was ended by bankruptcy: the bankruptcy of 1607 which sealed the fate of the Spanish Ausburghs in their fight against France, the bankruptcy of France in 1788 -caused by the war with Great Britain- which paved the way for the French revolution and the British dominance in the next hundred years, and the bankruptcy of the USSR in the late eighties of the past century -caused by the military expenses- that led to the collapse of the socialist block.

2. The role of chance. The importance of random events in conflicts cannot be underestimated. Clausewitz (1832) devoted the seventh chapter of his book to highlighting the influence of "frictions" on the outcome of war. Also there is a sizeable literature of contest in which the CSF arises as a reduced form of the effort of players and a random variable (Hillman and Riley 1989, Lazear and Rosen 1981, Dixit 1987, Fullerton and McAfee 1999, Baye and Hoppe 2003 and Jia 2007). In our case, a possible way to introduce random events would be by making the parameter $b$ a random variable.

We plan to study these aspects in the near future.

\section{Appendix}

\subsection{Second order conditions}

First we show that the second order conditions of profit maximization hold. Indeed,

$$
\begin{aligned}
\frac{\partial^{2} \Pi_{i}}{\partial e_{1}^{2}}= & \frac{\partial^{2} p}{\partial e_{1}^{2}} V\left[1+\delta f^{\prime}(p)(1-\gamma+2 \gamma f(p))\right]+\left(\frac{\partial p}{\partial e_{1}}\right)^{2} V \delta 2 \gamma\left(f^{\prime}(p)\right)^{2}+ \\
& +\left(\frac{\partial p}{\partial e_{1}}\right)^{2} V \delta f^{\prime \prime}(p)(1-\gamma+2 \gamma f(p))
\end{aligned}
$$

Note that,

$$
\frac{\partial p}{\partial e_{1}}=\frac{\gamma \alpha(1-\alpha) e_{1}^{\gamma-1} e_{2}^{\gamma}}{\left(\alpha e_{1}^{\gamma}+(1-\alpha) e_{2}^{\gamma}\right)^{2}}
$$

which is positive, and

$$
\frac{\partial^{2} p}{\partial e_{1}^{2}}=-\frac{\gamma \alpha(1-\alpha) e_{1}^{\gamma-2} e_{2}^{\gamma}}{\left(\alpha e_{1}^{\gamma}+(1-\alpha) e_{2}^{\gamma}\right)^{3}}\left(e_{2}^{\gamma}(1-\alpha)(1-\gamma)+e_{1}^{\gamma} \alpha(1+\gamma)\right)
$$

which is negative.

We show first that $\partial^{2} p / \partial e_{1}^{2}+2\left(\partial p / \partial e_{1}\right)^{2} \leq 0$. Note that $\partial^{2} p / \partial e_{1}^{2}+2\left(\partial p / \partial e_{1}\right)^{2}$ can be written as:

$$
\frac{\gamma \alpha(1-\alpha) e_{1}^{\gamma-2} e_{2}^{\gamma}}{\left(\alpha e_{1}^{\gamma}+(1-\alpha) e_{2}^{\gamma}\right)^{4}}\left[2 e_{1}^{\gamma} e_{2}^{\gamma} \gamma \alpha(1-\alpha)-\left(e_{2}^{\gamma}(1-\alpha)(1-\gamma)+e_{1}^{\gamma} \alpha(1+\gamma)\right)\left(\alpha e_{1}^{\gamma}+(1-\alpha) e_{2}^{\gamma}\right)\right] .
$$


Note that the term in brackets can be rewritten as:

$$
2 e_{1}^{\gamma} e_{2}^{\gamma} \alpha(1-\alpha)(\gamma-1)-e_{2}^{2 \gamma}(1-\alpha)^{2}(1-\gamma)-e_{1}^{2 \gamma} \alpha^{2}(1+\gamma)
$$

which is negative because $\gamma \leq 1$. Thus, $\partial^{2} p / \partial e_{1}^{2}+2\left(\partial p / \partial e_{1}\right)^{2} \leq 0$.

Note that since $f^{\prime \prime}(p) \leq 0$, and $\gamma \leq 1$, the last term in (6.1) is less or equal than zero. Since $f^{\prime}(p) \geq 0$, and $\partial^{2} p / \partial e_{1}^{2} \leq 0$, the first term is less or equal to $V\left(\partial^{2} p / \partial e_{1}^{2}\right)$, and since $f^{\prime}(p) \leq 1, \delta \leq 1$, and $\gamma \leq 1$, $\partial p / \partial e_{1}>0$, the second term is less or equal to $2 V\left(\partial p / \partial e_{1}\right)^{2}$. Finally, since $\partial^{2} p / \partial e_{1}^{2}+2\left(\partial p / \partial e_{1}\right)^{2} \leq 0$ we obtain that $\partial^{2} \Pi_{i} / \partial e_{1}^{2} \leq 0$, as we wanted to show.

\subsection{Existence of $x(\alpha)$}

Existence: Recall that $x(\alpha)$ is defined as the solution of

$$
1+\delta f^{\prime}(p)(1-\gamma+2 \gamma f(p))=x\left[1+\delta f^{\prime}(p)(1+\gamma-2 \gamma f(p))\right]
$$

Suppose $x \rightarrow 0$. Then, the left hand side is larger than the right hand side (which tends to zero). But if $x \rightarrow \infty$ the right hand side tends to infinite (note that, because of the assumptions on the transition function, the term in brackets is bounded) and is larger than the left hand side which tends to a positive real number. By the intermediate value theorem there is an $x$ such that both sides are identical, so (6.7) has indeed a solution.

\subsection{Proof of Proposition 2}

Existence of equilibrium is guarantee as we proved in the last section. We show that in the case of $\gamma=1$, and a linear transition function the equilibrium is unique. For that it is enough to show that the solution to $h_{1}(x, \alpha)-x h_{2}(x, \alpha)=0$ is unique. For $\gamma=1$, and a linear transition function, $h_{1}(x, \alpha)-x h_{2}(x, \alpha)=0$ can be written as:

$$
1+\delta 2 a\left(a \frac{\alpha x}{\alpha x+(1-\alpha)}+b\right)=x\left(1+\delta 2 a\left(1-a \frac{\alpha x}{\alpha x+(1-\alpha)}-b\right)\right) .
$$

Write (6.8) as follows

$$
1+\delta 2 a^{2} \frac{\alpha x}{\alpha x+(1-\alpha)}(x+1)+\delta 2 a b=x(1+2 \delta a-2 \delta a b) .
$$


The right hand side of (6.9) is linear and increasing, being zero when $x=0$. The left hand side of (6.9) takes a positive value when $x=0$. Furthermore, when $\alpha>1 / 2$ it is strictly concave. A linear function and a strictly concave function can intersect, at most twice. But given the behavior of both functions at $x=0$ the intersection is unique. If $\alpha \leq 1 / 2$ the left hand side of (6.9) is convex (linear if $\alpha=1 / 2$ ), thus the slope of the curve is increasing with $x$. When $x$ tends to infinity the slope tends to $\delta 2 a^{2}$. But notice that since $a+b \leq 1,1-2 \delta a b+2 \delta a \geq 1+\delta 2 a^{2}$, which implies that $\delta 2 a^{2}<1-2 \delta a b+2 \delta a$ thus the slope of the convex curve is always smaller than the slope of the linear function. Thus, given the behavior of both functions at $x=0$, the linear function and the convex function intersect just once.

\subsection{Proof of Proposition 3}

(i) If the strength of player 1 is $1-\alpha$, the first order conditions of the maximization problem for each player can be written as:

$$
\begin{aligned}
& \frac{\alpha(1-\alpha) e_{2}}{\left((1-\alpha) e_{1}+\alpha e_{2}\right)^{2}} V g_{1}(y, 1-\alpha)=1 ; \\
& \frac{\alpha(1-\alpha) e_{1}}{\left((1-\alpha) e_{1}+\alpha e_{2}\right)^{2}} V g_{2}(y, 1-\alpha)=1,
\end{aligned}
$$

where $y=e_{2} / e_{1}$, and

$$
\begin{gathered}
g_{1}(y, 1-\alpha)=1+\delta 2 a(a p+b), \\
g_{2}(y, 1-\alpha)=1+\delta 2 a(a(1-p)+b), \\
p=\frac{(1-\alpha)}{(1-\alpha)+\alpha y} ; 1-p=\frac{\alpha y}{(1-\alpha)+\alpha y} .
\end{gathered}
$$

Thus, from (6.10), and (6.11) we get that

$$
y g_{1}(y, 1-\alpha)-g_{2}(y, 1-\alpha)=0
$$

Notice that $g_{1}(y, 1-\alpha)=h_{2}(y, \alpha)$, and $g_{2}(y, 1-\alpha)=h_{1}(y, \alpha)$, thus equation (6.15) is identical to equation (3.16), which implies that

$$
y(1-\alpha)=x(\alpha)
$$


Thus, from (6.10) and the definition of $y$ we obtain that

$$
\begin{aligned}
e_{1}(1-\alpha) & =\frac{\alpha(1-\alpha) y(1-\alpha)}{((1-\alpha)+\alpha y(1-\alpha))^{2}} V g_{1}(y, 1-\alpha)= \\
& =\frac{\alpha(1-\alpha) x(\alpha)}{((1-\alpha)+\alpha x(\alpha))^{2}} V h_{2}(x, \alpha)= \\
& =\frac{\alpha(1-\alpha)}{((1-\alpha)+\alpha x(\alpha))^{2}} V h_{1}(x, \alpha)=e_{2}(\alpha),
\end{aligned}
$$

where we have made use of the fact that $g_{1}(y, 1-\alpha)=h_{2}(y, \alpha)$ and (3.16).

(ii) Trivially, if $\alpha=1$, or $\alpha=0, e_{1}=e_{2}=0$. And since $e_{2}(\alpha)=e_{1}(1-\alpha), e_{1}(1 / 2)=e_{2}(1 / 2)$. Thus $x(1 / 2)=1$.

(iii) We finally show that when $\alpha>1 / 2, x(\alpha)>1$ which implies that $e_{1}(\alpha)>e_{2}(\alpha)$. Recall that $x(\alpha)$ is the solution of $0=h_{1}(x, \alpha)-x h_{2}(x, \alpha)$. Since $h_{1}(x, \alpha)$ is increasing in $\alpha, h_{2}(x, \alpha)$ is decreasing in $\alpha$, and $\alpha>1 / 2$, we have that $h_{1}(x, \alpha)-x h_{2}(x, \alpha)>h_{1}(x, 1 / 2)-x h_{2}(x, 1 / 2)$. Since $x(1 / 2)=1, h_{1}(1,1 / 2)-h_{2}(1,1 / 2)=0$. Thus, $h_{1}(x, 1 / 2)-x h_{2}(x, 1 / 2)<h_{1}(1,1 / 2)-h_{2}(1,1 / 2)$. But note that $h_{1}(x, 1 / 2)-x h_{2}(x, 1 / 2)$ can be written as

$$
1+2 \delta a^{2} \frac{x}{x+1}+2 \delta a b-x\left(1+2 \delta a-2 \delta a^{2} \frac{x}{x+1}-2 \delta a b\right) .
$$

Rearranging terms,

$$
1+2 \delta a b+x(2 \delta a(a+b-1)-1)
$$

which is decreasing in $x$ because $a+b \leq 1$. Therefore, $h_{1}(x, 1 / 2)-x h_{2}(x, 1 / 2)$ is decreasing in $x$. Thus, $x(\alpha)>1$ for $\alpha>1 / 2$.

\subsection{Proof of Proposition 4}

Since $x(\alpha)$ is given by $h_{1}(x, \alpha)-x h_{2}(x, \alpha)=0$,

$$
x^{\prime}(\alpha)=\frac{-\frac{\partial h_{1}}{\partial \alpha}+x \frac{\partial h_{2}}{\partial \alpha}}{\frac{\partial h_{1}}{\partial x}-h_{2}-x \frac{\partial h_{2}}{\partial x}} .
$$

The sign of $\partial h_{1} / \partial \alpha$ depends on the sign of $\partial p / \partial \alpha$ which is positive. The sign of $\partial h_{2} / \partial \alpha$ depends on the sign of $-\partial p / \partial \alpha$ which is negative. Thus, the numerator in (6.22) is negative. We show next that the denominator is also negative. Note first that the denominator can be written as:

$$
\left.2 \delta a^{2} \frac{\partial p}{\partial x}-1-2 \delta a(a(1-p)+b)\right)+x 2 \delta a^{2} \frac{\partial p}{\partial x} .
$$


(6.23) can be rewritten as:

$$
2 \delta a^{2}\left(\frac{\partial p}{\partial x}(1+x)-(1-p)-1-2 \delta a b\right.
$$

Since $\partial p / \partial x=(\alpha(1-\alpha)) /(\alpha x+(1-\alpha))^{2}(6.24)$ can be rewritten as

$$
2 \delta a^{2} \frac{1-\alpha}{\alpha x+(1-\alpha)}\left(\frac{\alpha(1+x)}{\alpha x+(1-\alpha)}-1\right)-1-2 \delta a b .
$$

Simplifying (6.25) we obtain

$$
2 \delta a^{2} \frac{1-\alpha}{\alpha x+(1-\alpha)}\left(\frac{2 \alpha-1}{\alpha x+(1-\alpha)}\right)-1-2 \delta a b .
$$

Since the expression in brackets is negative for $\alpha \leq 1 / 2,(6.25)$ is negative as we wanted to prove. We show next that this is also the case for $a>1 / 2$. If $\alpha>1 / 2, x(\alpha)>1$ and since (6.26) is decreasing in $x$ it is smaller than

$$
2 \delta a^{2}(1-\alpha)(2 \alpha-1)-1-2 \delta a b
$$

which has a maximum at $\alpha=3 / 4$ then $(6.27)$ is smaller than

$$
\frac{\delta a^{2}}{4}-1-2 \delta a b
$$

which is always negative.

\subsection{Proof of Proposition 5}

Recall that $e_{i}^{o s}$ denotes the equilibrium effort of player $i$ in the one shot game. Note that in the one shot game both agents spend the same effort and $e_{1}^{o s}=e_{2}^{o s}=\alpha(1-\alpha) V$. We show that there exists $\alpha^{*}<1 / 2$ such that for all $\alpha \in\left(0, \alpha^{*}\right), e_{1}(\alpha)<e_{1}^{o s}(\alpha)$. Note first that by (3.17) the equilibrium effort of player 1 can be written as:

$$
e_{1}(\alpha)=e_{1}^{o s}(\alpha) \frac{x(\alpha)}{(\alpha x(\alpha)+(1-\alpha))^{2}} h_{1}(x(\alpha), \alpha)
$$

Let us see that there exists a unique $\alpha^{*}<1 / 2$ such that

$$
\frac{x(\alpha)}{(\alpha x(\alpha)+(1-\alpha))^{2}} h_{1}(x(\alpha), \alpha)=1
$$

For $\alpha=1 / 2, x(\alpha)=1$ and therefore $(6.30)$ is equal to $h_{1}(x(\alpha), \alpha)$. Recall that $h_{1}(x(\alpha), \alpha)=$ $1+\delta 2 a(a p+b)>1$. Thus, for $\alpha=1 / 2$ the left hand side of (6.30) is bigger than 1 . When $\alpha$ is close 
to zero, the left hand side of (6.30) is close to zero. Thus, by the intermediate value theorem there exists $\alpha^{*}<1 / 2$ such that equation (6.30) is satisfied. We show that the left hand side of equation (6.30) is strictly increasing for all $\alpha \leq 1 / 2$ which guarantees that $\alpha^{*}$ is unique. Since $h_{1}(x(\alpha), \alpha)$ is strictly increasing, it only remains to be proved that $x(\alpha) /(\alpha x(\alpha)+(1-\alpha))^{2}$ is increasing for all $\alpha \leq 1 / 2$. The first derivative of $x(\alpha) /(\alpha x(\alpha)+(1-\alpha))^{2}$ can be written as:

$$
\frac{x^{\prime}(\alpha)(-\alpha x(\alpha)+(1-\alpha))+2 x(\alpha)(1-x(\alpha))}{(\alpha x(\alpha)+(1-\alpha))^{3}} .
$$

Since $\alpha<1 / 2, x(\alpha)<1$, and $(1-\alpha)>\alpha>\alpha x(\alpha)$. Thus, (6.31) is positive as we wanted to show. Thus, there exists a unique $\alpha^{*}<1 / 2$ such that for all $\alpha \in\left(0, \alpha^{*}\right), e_{1}(\alpha)<e_{1}^{o s}(\alpha)$.

\subsection{Proof of Proposition 6}

We first recall the equations that we will use here, namely:

$$
\begin{aligned}
\alpha^{\prime} & =a p+b, \\
p & =\frac{\alpha x}{\alpha x+1-\alpha}, \\
x & =\frac{1+2 \delta a(a p+b)}{1+2 \delta a(1-a p-b)}, \\
a & =1-2 b .
\end{aligned}
$$

Using (6.32), equations (6.33) and (6.34) can be written as

$$
\begin{aligned}
\alpha x\left(\alpha^{\prime}-b-a\right) & =\left(b-\alpha^{\prime}\right)(1-\alpha) . \\
x & =\frac{1+2 \delta a \alpha^{\prime}}{1+2 \delta a\left(1-\alpha^{\prime}\right)} .
\end{aligned}
$$

Substituting the value of $x$ in (6.37) in equation (6.36) we obtain that

$$
\alpha\left(\alpha^{\prime}-b-a\right)\left(1+2 \delta a \alpha^{\prime}\right)-\left(b-\alpha^{\prime}\right)(1-\alpha)\left(1+2 \delta a\left(1-\alpha^{\prime}\right)\right)=0
$$

which will be our main equation in this proof.

Our first step is to study the roots of (6.38) when $\alpha=\alpha^{\prime}$. Notice that in this case (6.38) is a cubic function of $\alpha$

$$
\alpha(\alpha-b-a)(1+2 \delta a \alpha)-(b-\alpha)(1-\alpha)(1+2 \delta a(1-\alpha))=0 .
$$

Note that $\alpha=1 / 2$ is always a solution of (6.39), and if $\bar{\alpha}$ is a solution of (6.39), then $1-\bar{\alpha}$ is also a solution of (6.39). Also note that (6.39) can be written as

$$
4 \delta(1-2 b) \alpha^{3}+6 \delta(2 b-1) \alpha^{2}+2\left(\delta-4 \delta b^{2}+b\right) \alpha+b(4 b \delta-1-2 \delta)=0 .
$$


By using numerical methods it can be shown that (6.40) has, at most, three solutions in $\alpha$.

Our second step is to compute $d \alpha^{\prime} / d \alpha$. Let us call the left hand side of (6.38) $F\left(\alpha, \alpha^{\prime}\right)$. Totally differentiating (6.38) we obtain that

$$
\frac{d \alpha^{\prime}}{d \alpha}=\frac{-\frac{\partial F\left(\alpha, \alpha^{\prime}\right)}{\partial \alpha}}{\frac{\partial F\left(\alpha, \alpha^{\prime}\right)}{\partial \alpha^{\prime}}}
$$

We now compute

$$
-\frac{\partial F\left(\alpha, \alpha^{\prime}\right)}{\partial \alpha}=\left(1+2 \delta a \alpha^{\prime}\right)\left(a+b-\alpha^{\prime}\right)+\left(\alpha^{\prime}-b\right)\left(1+2 \delta a\left(1-\alpha^{\prime}\right)\right) .
$$

Since $\alpha^{\prime}>b$ (from (6.32)) and $\alpha^{\prime}<b+a$ (from (6.36)), both terms in the right hand side of (6.42) are positive.

Let us now study the denominator of (6.41). We compute

$$
\frac{\partial F\left(\alpha, \alpha^{\prime}\right)}{\partial \alpha^{\prime}}=2 \delta a\left(4 \alpha \alpha^{\prime}-2 \alpha-2 \alpha^{\prime}+1+b\right)+1
$$

Thus, using (6.42) and (6.43), (6.41) can be written as

$$
\frac{d \alpha^{\prime}}{d \alpha}=\frac{\left(1+2 \delta a \alpha^{\prime}\right)\left(1-b-\alpha^{\prime}\right)+\left(\alpha^{\prime}-b\right)\left(1+2 \delta a\left(1-\alpha^{\prime}\right)\right)}{2 \delta a\left(4 \alpha \alpha^{\prime}-2 \alpha-2 \alpha^{\prime}+1+b\right)+1} .
$$

Next we compute $d \alpha^{\prime} / d \alpha$ evaluated at $\alpha=\alpha^{\prime}=1 / 2$ which amounts to

$$
\frac{d \alpha^{\prime}}{d \alpha}=\frac{2(1+\delta a)(1 / 2-b)}{2 \delta a b+1} .
$$

Rearranging the previous expression we obtain that

$$
\begin{gathered}
\frac{d \alpha^{\prime}}{d \alpha}>1 \text { if and only if } \delta>\frac{2 b}{(1-2 b)(1-4 b)} \text { and } b<1 / 4 \\
\frac{d \alpha^{\prime}}{d \alpha}<1 \text { if and only if } \delta<\frac{2 b}{(1-2 b)(1-4 b)} \text { and } b<1 / 4 \text {, or } b \geq 1 / 4 \text { and } \delta \in[0,1] .
\end{gathered}
$$

Finally when $\alpha=0$, equation (6.38) which defines $\alpha^{\prime}$ as a function of $\alpha$ is $-\left(b-\alpha^{\prime}\right)\left(1+2 \delta a\left(1-\alpha^{\prime}\right)\right)=$ 0 . This equation has only one root $\alpha^{\prime}=b$.

Now we have all the necessary ingredients to prove the proposition. We will do it for the case $\alpha<1 / 2$. The case $\alpha>1 / 2$ is totally symmetric.

Part a). If $b=0, d \alpha^{\prime} / d \alpha>1$ so the curve relating $\alpha$ with $\alpha^{\prime}$ cuts the $45^{0}$ degree line from below. In this case (6.39) has three solutions in alpha, namely $0,1 / 2$ and 1 . Given the geometry of the problem, the avalanche effect occurs for all $\alpha \in[0,1] \backslash\{1 / 2\}$. 
Part b). If $\delta>2 b /(1-2 b)(1-4 b)$ and $b<1 / 4, d \alpha^{\prime} / d \alpha>1$. Since when $\alpha<1 / 2$, but sufficiently close to $1 / 2, \alpha^{\prime}<\alpha$ and when $\alpha=0, \alpha^{\prime}=b$, by continuity the function relating $\alpha$ to $\alpha^{\prime}$ must cut the $45^{0}$ line so the existence of $\bar{\alpha}$ is guaranteed. The symmetry of the function around $1 / 2$ and the existence of at most three solutions to (6.40) imply that this intersection is unique in $(0,1 / 2)$. Thus for all $\alpha \in(0, \bar{\alpha})$ there is a level-off effect and for $\alpha \in(\bar{\alpha}, 1 / 2)$ there is an avalanche effect. Part c). If $b<1 / 4$ and $\delta<2 b /(1-2 b)(1-4 b)$, or if $b \geq 1 / 4$ and $\delta \in[0,1], d \alpha^{\prime} / d \alpha<1$. Thus when $\alpha$ is less than $1 / 2$, but sufficiently close to $1 / 2, \alpha^{\prime}>\alpha$ and when $\alpha=0, \alpha^{\prime}=b$. The function relating $\alpha$ and $\alpha^{\prime}$ does not fall below the $45^{0}$. There might be a point at which $\alpha=\alpha^{\prime}$ but just one because if this function cuts twice the $45^{0}$, by symmetry, there would be 5 solutions to (6.40) which is impossible. Thus the level-off effect holds for all $\alpha \in[0,1]$ except, possibly, for two values of $\alpha$. 


\section{References}

[1] Baye, M. R. and H. C. Hoppe. "The Strategic Equivalence of Rent-Seeking, Innovation, and Patent-Race Games". Games and Economic Behavior 44, 217-226, 2003.

[2] Becker, G. "A Theory of Competition Among Pressure Groups for Political Influence". The Quarterly Journal of Economics, 98, 371-400, 1983.

[3] Cairns, R. "Dynamic Rent Seeking". Journal of Public Economics 39, 315-334, 1989.

[4] Clarke D, Riis C. Contest success functions: an extension. Economic Theory, 11, 201-204, 1998.

[5] Clausewitz, C. von. On War. 1832. Spanish edition translated by Carlos Fortea. La Esfera de los Libros, Madrid, 2005.

[6] Corchón, L. C. "On the Allocative Effects of Rent-Seeking". Journal of Public Economic Theory, 2, n. 4, 483-491, October 2000.

[7] Cornes, R. and R. Hartley. "Asymmetric contests with general technologies". Economic Theory, 26, 4, 923-946, 2005.

[8] Dixit, A. K. "Strategic Behavior in Contests". The American Economic Review, 77, 5, 891-898, 1987.

[9] Fearon, J. D. 1996. "Bargaining Over Objects That Influence Future Bargaining Power." Presented at the annual meeting of the American Political Science Association, Washington

[10] Franke, J., C. Kanzow, W. Leininger and A. Väth. "Effort Maximization in Asymmetric N-person Contest Games," Ruhr Economic Papers 0130, Rheinisch-Westfälisches Institut für Wirtschaftsforschung, Ruhr-Universität Bochum, Universität Dortmund, Universität Duisburg-Essen, 2009.

[11] Fullerton, R. L. and R. P. McAfee. "Auctioning Entry into Tournaments". Journal of Political Economy, 107, 573-605, 1999.

[12] Garfinkel, M. R. and S. Skaperdas. "Conflict Without Misperceptions or Incomplete Information: How the Future Matters". Journal of Conflict Resolution, 44, 6, 793-807, 2000. 
[13] Gradstein, M. "Intensity of Competition: Entry and Entry Deterrence in Rent-Seeking Contests". Economics and Politics, 7, March 1995.

[14] Hillman, A. and J. Riley. "Politically Contestable Rents and Transfers". Economics and Politics, 1, 1, 17-39, 1989.

[15] Hirshleifer, J. "The Technology of Conflict as an Economic Activity". The American Economic Review, 81, 2, Papers and Proceedings, 130-134. 1991

[16] Jia, H. (2007), "A Stochastic Derivation of Contest Success Functions". Public Choice, 135, 3-4, 125-130, 2008.

[17] Klumpp, T. and M. Polborn. "Primaries and the New Hampshire Effect". Journal of Public Economics 90, 1073-1114, 2006.

[18] Konrad, K. Strategy and Dynamics in Contests. Oxford University Press, 2009.

[19] Konrad, K. and D. Kovenock. "Multi-Battle Contests". Games and Economic Behavior, 66, 1, 256-274, 2009.

[20] Konrad, K. and D. Kovenock. "Contests with Stochastic Abilities". Economic Inquiry, 48, 1, 89-103, 2010.

[21] Konrad, K. "Dynamic Contests" Max Planck Institute for Intellectual Property, Competition and Tax Law and WZB August 2010.

[22] Krueger, A. "The Political Economy of the Rent-Seeking Society". American Economic Review, 64, 291-303, 1974.

[23] Lazear, E. P. and S. Rosen. "Rank-Order Tournaments and Optimum Labor Contracts". The Journal of Political Economy, 89, 5, 841-864, 1981.

[24] Leininger W. and Y. Chun-Lei. "Dynamic Rent-Seeking Games". Games and Economic Behavior, 7, 3, 406-427, 1994.

[25] Leventoglu, B. and B. L. Slantchev. "The Armed Peace: A Punctuated Equilibrium Theory of War". American Journal of Political Science, 51, 4, 755-771, 2007. 
[26] McBride, M. and S. Skaperdas. "Explaining Conflict in Low-Income Countries: Incomplete Contracting in the Shadow of the Future," in M. Gradstein and K. A. Konrad, (eds.), Institutions and Norms in Economic Development, 141-161, 2007, Cambridge, MA: MIT Press.

[27] Nti, K. "Maximum efforts in contests with asymmetric valuations". European Journal of Political Economy, 20 1059-1066, 2004.

[28] Shapiro, C. "Theories of Oligopoly Behavior". In R. Schmalensee \& R. Willig (eds.) Handbook of Industrial Organization, chapter 6, 329-414, 1989.

[29] Skaperdas, S. "Contest Success Functions". Economic Theory 7, 283-290, 1996.

[30] Skaperdas, S. and C. Syropoulos. "Can the Shadow of the Future Harm Cooperation?". Journal of Economic Behavior and Organization, May 1996, 29, 355-372.

[31] Tullock, G. "The Welfare Cost of Tariffs, Monopolies and Theft". Western Economic Journal, 5, pp. 224-232, 1967.

[32] Tullock, G. (1980). "Efficient Rent Seeking". In J. Buchanan, R. Tollison, and G. Tullock (eds.), Toward a theory of the rent seeking society. College Station: Texas A\&M University Press, 97-112, 1980.

[33] Wirl, F. "The dynamics of lobbying, A differential game". Public Choice 80, 307-323, 1994. 